\title{
Evolving adoptive cellular therapies in urological
}

\section{malignancies}

Yien Ning Sophia Wong, MD ${ }^{1.2,3 *}$, Kroopa Joshi, MD ${ }^{2,4 *}$, Martin Pule, MD,PhD ${ }^{2,5}$, Professor Karl S Peggs, MD, $\mathrm{PhD}^{2,5}$, Professor Charles Swanton, MD,PhD,FMedSci ${ }^{1,3,6,7}$, Sergio A. Quezada, $\mathrm{PhD}^{2}$, Mark Linch, MD, $\mathrm{PhD}^{1,3 \wedge}$

${ }^{*}$ These authors contributed equally to this work.

${ }^{\wedge}$ Corresponding author

\section{Affiliations}

1. Department of Oncology, UCL Cancer Institute, 72 Huntley Street, London, WC1E 6BT, UK.

2. Immune Regulation and Tumour Immunotherapy Laboratory, UCL Cancer Institute, 72 Huntley Street, London, WC1E 6BT, UK.

3. Department of Oncology, University College London Hospital, 250 Euston Rd, London, NW1 2PG, UK.

4. Department of Medical Oncology, Royal Marsden Hospital, Fulham Road, London, SW3 6JJ, UK.

5. Department of Haematology, University College London Hospitals, 250 Euston Rd, London, NW1 2PG, UK.

6. Translational Cancer Therapeutics Laboratory, UCL Cancer Institute, 72 Huntley Street, London, WC1E 6BT, UK.

7. Translational Cancer Therapeutics Laboratory, The Francis Crick Institute, 1 Midland Rd, London NW1 1AT, UK. 


\section{SUMMARY}

Immunotherapies have long been used to treat urological cancers but rarely lead to cure. Recent success of immune checkpoint inhibition has led to a resurgence of enthusiasm for immunotherapy in solid tumours. Increased understanding of tumour immune biology, technological advancements of gene transfer and cell culture as well improved clinical infrastructures for routine delivery of cell products has made cell based immunotherapies an enticing and real prospect. These scientific and clinical activities, attempting to exploit the innate and adaptive immune systems for therapeutic gain, are well exemplified by the urological malignancies of renal, bladder, prostate and penile cancer, a group of anatomically localised diseases, each with distinct biology and therefore different immunotherapeutic challenges. Results of clinical studies of autologous cellular therapies in urological malignancies are presented, along with the rationale for upcoming studies, and how novel therapies and adoptive cell combinations can be utilised for personalised cancer therapy. 


\section{INTRODUCTION}

The immune system has evolved to protect the host from infections and from cancer. Following the recent discovery of therapeutically beneficial immune checkpoint inhibitors, there is a real belief that immunotherapy will be transformative for cancer. Immune mediated treatment strategies may be considered as cell based or non-cell based with cell based strategies being developed to exploit the main immune cell repertoires (Table 1)

Harnessing the anti-cancer activity of the immune system has been an ambition since the 1890s, when William Coley witnessed a patient with sarcoma have a complete remission following Streptococcus pyogenes infection. Coley went on to use bacterial innoculations to treat cancer patients. ${ }^{1}$ leading to the use of Calmette-Guerin (BCG) as a cancer immunotherapy, which remains a standard of care for superficial bladder today. The first report of successful cell based (adoptive cell therapy, ACT) in humans was nearly 100 years later where administration of autologous lymphokine activated killer cells (non-T, non-B cells extracted from peripheral blood) demonstrated notable tumour responses. ${ }^{2}$ Most recently, it has been demonstrated in a patient with colorectal cancer that ex-vivo expansion and re-infusion of their KRAS G12D mutant CD8+ T cells can lead to clinically significant remission of metastatic disease. ${ }^{3}$

The use and development of cell based adoptive immunotherapies has gained traction as a result of 3 main factors: 1) significant insights into the biology of immune oncology, 2) the ability to enrich for and expand specific immune cells and 3) development of an infrastructure for the delivery of cellular products to patients in a reproducible and quality assured manner. For this review of cell based therapies, we have selected urological malignancies as an exemplar - in particular, prostate, bladder, renal and penile cancer. We will describe the new biological insights that are driving the development of cellular therapy for each tumour type and present the resulting clinical investigations and forward projections.

\section{INNATE IMMUNITY}

\section{Dendritic cells}


Dendritic cells may be considered as natural adjuvants to anti-tumour immunity (for review see ${ }^{4}$ ). They represent approximately $0.4 \%$ of peripheral blood mononuclear cells, ${ }^{5}$ and are divided into two major subsets: myeloid (often referred to as conventional DCs) and plasmacytoid based on their tissue expression and cell surface markers. Immature DCs are able to take up exogenous antigens from blood and tissue via a number of processes including $\mathrm{Fc} \gamma$ receptor III mediated uptake, macropinocytosis and mannose receptor-mediated uptake. Following antigen capture the DCs migrate to the draining lymph nodes, and in the presence of infection or necrosis they mature to cells equipped for T-cell activation, upregulating major histocompatibility factor (MHC) and co-signaling molecules such as CD40 and B7. Three main strategies have been applied to utilize DCs as anticancer therapies, exvivo antigenic peptide loading followed by autologous infusion of the conditioned DCs, gene modification of dendritic in vivo through the use of recombinant viruses or ex-vivo genetic engineering for antigen presentation with or without enhanced co-signaling. Here we focus on the antigen/peptide loaded dendritic cell therapy as this is the most clinically developed in urological malignancies.

\section{Dendritic cell therapies}

The most studied dendritic cell therapy is sipuleucel-T, that involves the modification of autologous dendritic cells from peripheral blood monocytes (PBMC) by ex-vivo pulsation with GM-CSF linked prostatic acid phosphatase peptide, a tumour-derived peptide. The stimulated cells are then re-infused back into the patient where they activate anti-tumour $\mathrm{T}$ cells and promote tumour cell killing. The double blind, randomised phase III clinical trial, in 512 patients with metastatic castration-resistant prostate cancer (mCRPC) demonstrated a 4.1 months benefit in overall survival with manageable toxicities of fever, headache and chills. ${ }^{6}$ The Food and Drug Administration (FDA) approval of sipuleucel-T followed in 2010, the first adoptive cell therapy approved for any type of cancer. However, sipuleucel-T has not been universally adopted due to difficulties in routine manufacture of the product, concerns about possible negative impact of the repeated leukapheresis alone in the control arm of the study and that it failed to meet the studies original co-primary endpoint of improved time to objective disease progression and time to disease related pain. However, lack of progression free survival (PFS) despite overall survival (OS) benefit has now been replicated in other immunotherapy studies, ${ }^{7}$ and may be due in part to significantly delayed treatment responses and difficulties in accurately evaluating radiological progression in prostate cancer. As a result, sipuleucel-T is now under 
investigation in numerous combination trials in prostate cancer (see combination section).

Lapuleucel-T (DN24-02, Neuvenge) is also a dendritic cell based vaccine that has been tested for Her2 positive urothelial carcinoma using the same antigen delivery platform as for sipuleucel-T. The PBMCs are instead pulsed with GM-CSF linked recombinant Her2 peptide. Bladder cancer frequently has activating mutations of Her2 which confer poor clinical outcomes. ${ }^{8}$ A phase II trial of adjuvant lapuleucel-T therapy in high risk muscle invasive HER2/neu positive (HER2/neu tissue expression $\geq 1+$ by immunohistochemistry) urothelial bladder cancer failed to demonstrate a statistically significant difference in overall or disease free survival. However, patients with a lower disease burden and no prior neoadjuvant chemotherapy were found to have a trend towards improved survival. ${ }^{9}$

As an alternative to recombinant peptide loading, Podrazil et al pulsed immature autologous DCs with irradiated LNCaP prostate cancer cells (DCVAC/PCa), prior to maturation with poly $\mathrm{I}: \mathrm{C}$ and then subcutaneous administration to the patient. The injections were repeated up to 10 times, were preceded by 7 days of metronomic cyclophosphamide and initially co-administered with 6 cycles of docetaxel chemotherapy. This regimen led to a decrease in immunosuppressive regulatory Tcells (Treg) that may account for an overall survival well above historical controls (19 vs 11.8 months). ${ }^{10}$ This preparation is currently being tested in the phase III VIABLE study (NCT02111577).

In metastatic renal cancer (mRCC), there are several studies testing genetic modification of DCs ex-vivo before autologous infusion. In a phase 2 study of dendritic cells that were transfected with amplified tumour RNA and synthetic CD40L RNA in combination with sunitinib, 9/21 patients with intermediate to poor risk mRCC had a partial response. ${ }^{11}$ Notably in this study that closed to recruitment early due to insufficient complete responses, survival was associated with absolute effector/memory T-cell number after 5 cycles of treatment suggesting that immune modulation is governing the clinical outcome. Furthermore, a phase III trial of AGS003 (an autologous dendritic cell vaccine) combined with Sunitinib in patients with metastatic clear cell RCC has completed enrollment and results are awaited (NCT01582672). 
It is important however, to recognise that DC activation may not always result in antitumour immunity, indeed it has been shown that DC activation via the asialoglycoprotein receptor leads to the formation of IL-10 producing CD4+ suppressor cells which could in fact have a detrimental effect on the anti-tumour immune response. $^{12}$

Although not an adoptive therapy, the successes of in vivo vaccination strategies in urological malignancies supports the ongoing development of autologus dendritic cells. It is important therefore to briefly mention vaccine therapies giving an exemplar for each of prostate cancer, bladder cancer and renal cancer. ProstVAC is an engineered poxvirus containing PSA and three immune-enhancing co-stimulatory molecules, LFA-3, ICAM-1 and B7.1. The vaccine is injected subcutaneously into the patient, resulting in PSA epitope presentation on dendritic cells (and somatic cells) promoting an anti-tumour T-cell response. Updated analysis of the randomised phase II study conducted in 125 patients with mCRPC demonstrated a 9.9 month improvement in overall survival compared to the control (26.2 vs 16.3 months; HR 0.5, $p=0.0019$ ). This clinically relevant result has led to a phase III randomised study which has now completed accrual and the results are awaited. ${ }^{13}$ In $\mathrm{mRCC}$, phase I and II studies of IMA901, a multipeptide vaccine of tumour associated antigens, demonstrated good tolerability and in patients that mounted a CD8+ specific T-cell response to $\geq 1$ peptides there was an association with prolonged survival. ${ }^{14}$ Following on from the promising findings of this phase II study, a phase III study of vaccine in combination with sunitinib was performed but with disappointing results (see combination section). Similar to the IMA901 study, but in previously treated metastatic bladder cancer (mBC), S-288310, a HLA-A*24 restricted 2-peptide vaccine was tested as a monotherapy in 27 patients and led to a disease control rate of $56.3 \%$, a radiological partial response rate of $6.3 \%$ and a prolonged overall survival in patients that demonstrated T-cell induction to both peptides ${ }^{15}$. Given the activity signal of S288310 in $\mathrm{mBC}, 5$-peptides vaccines have been developed and are being tested in phase I/II studies and combination studies are planned.

\section{Identifying individualized neoantigens in improving dendritic cell therapies}

Tumour antigens that are shared between individuals are referred to as tumour associated antigens (TAAs). These are self-antigens that are differentially expressed in tumour compared to normal tissue but are also expressed in the thymus resulting in central tolerance; deletion of the highly reactive T-cell repertoire and development of 
suppressive Treg cells. A clue to how to circumvent this immune tolerance and potentially generate improved dendritic cell vaccination strategies has recently been informed by two separate strands of immunotherapy research - tumour infiltrating lymphocytes (TILs) and immune checkpoint blockade. In melanoma patients responding to TIL therapy, it was shown that there was a clonal expansion in individualized neoantigen reactive T-cells rather than the TAA reactive T-cells. Similarly, patients who were responding to anti-CTLA-4 or PD-1 inhibition were found to have high mutational burden, high neoepitope load and again expansion in neoantigen reactive T-cells. ${ }^{16-18}$ The clonal nature of somatic mutations impacts upon the clonality of neoantigens; mutations present in all tumour regions give rise to clonal neoantigens whereas mutations that are restricted to specific tumour regions give rise to sub-clonal neoantigens. The Swanton and Quezada laboratories have demonstrated, using clinical trial data sets, increased responsiveness to anti-CTLA-4 and anti-PD-1 in metastatic melanoma and NSCLC respectively in tumours enriched for clonal neoantigens. ${ }^{19}$

These discoveries were heavily reliant on a number of technological advancements. Firstly, this was only possible due to the ability to perform massively parallel sequencing on tumour samples. Secondly, the bioinformatics has developed such that using individual patients' tumour somatic nucleotide variation (SNV) datasets combined with the patient specific HLA genotype, it is possible to predict which neoantigens may be strongly bound to patient specific MHC class $\mathrm{I}^{17}$ Finally, in order to validate these predictions, assays have been optimised using recombinant peptides corresponding to the predicted neoantigens that are bound to patient specific synthetic MHC multimers. TILs are incubated with fluorescently labelled MHC/peptide multimers and can be sorted using flow cytometry. The TILs that are strongly bound to the $\mathrm{MHC} /$ peptide may be collected and undergo further functional validation. This approach is being taken forward by a number of commercial ventures, particularly to identify neoantigens suitable for dendritic therapy but also for selected TIL therapy (see TIL section). This individualised therapy clearly has economic and logistical hurdles and the bioinformatics predictions currently work less well for MHC class II epitopes. However, it is an exciting direction for immunotherapy that heralds much promise. 
Similar to dendritic cells, natural killer cells form an integral component of the innate immune system comprising about $10 \%$ of all lymphocytes in peripheral blood (for review see ${ }^{20}$ ). Primed NK cells are able to interact with and kill stressed cells, such as virally infected or tumour cells, without prior sensitisation. NK cell recognition occurs either by loss of inhibitory signals, such as MHC class 1 interaction with NK receptors, or tumour cell upregulation of NK-cell activating ligands such as haemagglutinin and cytomegalovirus. NK mediated killing may be via exocytosis induced cell lysis or Tumour Necrosis Factor (TNF) related apoptosis.

In order for NK cell function, there must be a priming signal, for example the interaction between CD2 on the NK-cell and its ligand CD15 on the tumour cell. ${ }^{21}$ Tumours cells that lack the CD15 priming molecule, such as the androgen resistant prostate cancer cell line DU145 may still undergo NK cell induced lysis if the NK cells are pre-incubated ex-vivo with a CD15+ tumour cell line, highlighting the importance of CD15 for NK cell anti-tumoural activity. This priming strategy was applied to NK-cells from haploidentical donors in a phase 1 study of 7 patients with Acute Myeloid Leukaemia. The study demonstrated good tolerability and led to 4/7 anti-leukaemic responses including 1 complete response lasting for more than 1 year. ${ }^{22}$ Studies utilising primed NK-cells in prostate cancer are planned. Furthermore, the role of bortezomib in combination with autologous NK adoptive cellular therapy is currently being investigated in patients with prostate cancer (NCT00720785). Of note, the rationale for this trial design is based on promising pre-clinical data demonstrating that bortezomib-induced upregulation of TNF related apoptosis induced ligand (TRAIL) receptor on the DU145 prostate cancer cell line led to enhanced NK-cell mediated cytotoxicity. ${ }^{23}$ In patients with advanced renal cancer, several phase I trials of ex vivo expanded allogeneic NK-92 cells, an immortalised NK-cell line, have already reported effective anti-tumour activity. ${ }^{24}$

\section{ADAPTIVE IMMUNITY}

The anti-tumour activity of adoptive cell therapy in human cancer was first recognised by Southam and colleagues who performed subcutaneous auto-transplants of advanced solid tumours with and without leukocytes. Inhibition of tumour growth was seen in half of the 41 patients co-inoculated with tumour and leucocytes. ${ }^{25}$ The transplantation field have helped to define that T-cells are key effectors of the antitumour activity, ${ }^{26}$ and the potential of T-cell therapy to have specific, deep and durable responses has led to great enthusiasm for adoptive T-cell therapy. There are two main 
strategies of adoptive T-cell therapy - use of native anti-tumour T-cells or lymphocytes engineered to target the tumour.

\section{Native Anti-tumour T-cells}

\section{Tumour infiltrating lymphocytes (TILs)}

Most cells, including cancer cells, use intracellular antigen presentation machinery to display self and non-self peptides bound to major histocompatibility complex 1 (MHC1) on their cell surface. Interaction between $\alpha \beta \mathrm{T}$ cell receptor on T-cells and MHC/nonself peptides along with a co-stimulatory signal leads to immune activation. Tumours are commonly infiltrated by $T$ cells directed against TAAs and neoantigens. Extraction of these TILs, ex-vivo expansion and re-infusion into the patients as an anti-cancer therapy (Figure 1), was first demonstrated at the National Cancer Institute in the 1980s. ${ }^{27}$ Since then, different lymphodepleting preconditioning regimens have been used between centres including non-myeloablative cyclophosphamide and fludarabine chemotherapy or the addition of total body irradiation as a myeloablative approach. Intravenous IL-2, at low, intermediate or high dose, is generally administered post TIL infusion, ${ }^{28}$ with a view to enhancing TIL proliferation, survival and effector function.

Effective ex-vivo expansion of the TIL population is critical for successful TIL reconstitution. The most important reagent for ex-vivo TIL expansion has been IL-2, but now a cocktail of activating factors are commonly employed. This includes the use of anti-CD3 (OKT3), ${ }^{29}$ anti-CD28, ${ }^{30}$ and allogeneic feeder cells such as irradiated PBMCs from healthy donors.

Once extracted, there is a balance between the number of expanded TILs needed for successful reconstitution and the effector activity that declines with successive passaging. TILs may be functionally selected based on their ability to induce cytokine production or tumour cell lysis following in vitro incubation with autologous tumour cells. More recently, a number of groups have selected TILs based on their ability to bind predicted tumour neoantigens, the so called neoantigen reactive T-cells. ${ }^{28}$

\section{Engineered Anti-tumour T cells}

In many situations, a strategy using native $T$ cells is not possible either due to insufficient biopsy material or more fundamentally due to lack of infiltrating 
lymphocytes within the tumour. To overcome these limitations, strategies that use genetic modification to confer specificity to effector cells have been developed. Here, peripheral blood T-cells are modified ex-vivo to express a receptor which re-directs them to a tumour antigen. There are two main strategies of genetic modification utilized (Figure 2): introduction of a gene coding for chimeric antigen receptor (CAR), which are antibody-based receptors, or introduction of genes coding for regular T-cell receptors. The genetic modification is typically achieved using an integrating vector such as a retroviral or lentiviral vector enabling a permanent modification that is propagated to the progeny of the cell product.

\section{Chimeric Antigen Receptor T cells (CARs)}

CARs graft the specificity of a monoclonal antibody (mAb) onto a T-cell. In a typical configuration, CARs are artificial type I transmembrane proteins with an aminoterminal extracellular domain, and carboxy-terminal intracellular domain. The extracellular domain contains an antigen binding domain, typically composed of a mAb derived single-chain variable fragment (scFv). The intracellular domain contains portions of T-cell signalling proteins. Hence, binding of the CAR to its cognate antigen results in activation of the T-cell. The main advantage of CARs over TCRs is that they recognize antigen in an HLA un-restricted fashion. ${ }^{31}$ In addition, CARs are not restricted to proteins but can recognize glycosylation variants and non-protein antigens providing they are expressed on the cell surface. ${ }^{32}$

Early CARs transmitted only an activation signal upon antigen recognition. These so called "first generation" CARs directed T-cell mediated killing, but CARs failed to proliferate and survive after antigen encounter. Perhaps unsurprisingly, clinical studies with first generation CARs were disappointing, characterized by low-level short-lived engraftment and fleeting responses. ${ }^{33,34}$ Incorporation of co-stimulatory signalling components along with activating signals resulted in CARs that transmitted killing, proliferation and survival signals to the expressing T-cells. ${ }^{35,36}$ These second- and third-generation CARs in contrast, have shown improved ability to engraft and expand within patients. ${ }^{37}$ When administered after lymphodepleting chemotherapy, $2^{\text {nd }}$ generation CARs directed against CD19 have shown remarkable activity in refractory B-cell cancers (for review see ${ }^{38}$ ) with durable responses that correlated with the persistence of CARs. ${ }^{39}$ CD19 CAR T-cell therapy will rapidly become the standard-ofcare in refractory B-cell malignancies, with licensed cell products expected this year for diffuse-large B-cell Lymphoma and paediatric B-ALL. 
CARs developments are also closely allied to more advanced cellular engineering which aim to improve the safety and performance of the engineered T-cell. ${ }^{40}$ These engineering strategies include; 1) suicide genes which allow selective depletion of CARs in the face of unacceptable toxicity, 2) payload release, where a cytokine payload such as IL-12 is selectively delivered into the tumour microenvironment, 3) coexpression of chemokine receptors that direct T-cell migration to the tumour bed, 4) Advanced CAR design that allows targeting of patterns of antigen expression increasing the ability to discriminate tumour from normal tissues and 5) split CAR designs that allow CAR T-cells to be "remotely controlled" by small molecule pharmaceuticals. In addition, genome editing technologies have been applied to CAR T-cells to knock out the endogenous TCRs, preventing the recognition of non-self antigens, enabling "off-the-shelf" allogenic CAR T-cells to be given without causing graft-versus host disease.

The next challenge for CAR T-cell therapy is to deliver efficacy in solid tumours. To date, no convincing clinical data exists in solid tumours, although relatively little clinical development has been performed in this area. Bringing CAR-T-cell therapy to urological malignancies requires overcoming several challenges. These include identification of suitable targets with minimal expression on normal tissue, the prevention of tumour immune escape and the facilitation of sufficient and persistent $T$ cell infiltration into the tumour microenvironment. ${ }^{33,41}$

\section{T Cell Receptor gene transfer}

TCRs direct the activity of T-cells to peptide/MHC complexes on target cells. Individual TCRs are composed of a single alpha chain linked to a beta chain by a disulphide bond, each with a variable and constant region. An alternative to engineering T-cells with genes coding for a CAR, is engineering with genes coding for a tumour-specific TCR. The main advantage of this approach is the ability to not only target membranebound antigen, but to also interrogate tumours for intracellular antigens. The main disadvantage is that TCRs are MHC-restricted and are typically developed targeting antigen in the context of HLA-A2 which makes this form of treatment available for approximately half of the population.

The generation of tumour specific TCRs has been achieved in several ways including immunisation of transgenic mice expressing HLA with peptides found in human 
cancers leading to a tumour antigen specific T cell response, ${ }^{42}$ identification of TCRs from patients, and by phage display. There have been some encouraging results of adoptive cellular therapy utilising $T$ cells harbouring genetically modified TCRs, but initial studies were 15 years ago and progress has been slow so far. ${ }^{43-45}$

\section{Prostate-specific membrane antigen}

Prostate-specific membrane antigen (PMSA; also known as glutamate carboxypeptidase 2 ) is a type II membrane protein most highly expressed on prostate cancer cells but also expressed in renal proximal tubule, type II astrocytes, and endothelial cells. ${ }^{46-48}$ Therefore, PSMA CAR T cells may have a dual anti-cancer action by directly targeting the prostate adenocarcinoma and the endothelium lined tumour vasculature. Two phase 1 trials of PSMA CAR-T cells have demonstrated tolerability and some activity (Table 2). The first study in 5 patients with mCRPC used a first generation PSMA CAR-T cell with chemotherapy pre-conditioning and continuous low dose IL-2. ${ }^{49}$ Two patients achieved a partial response with $50 \%$ and $70 \%$ serum prostate specific antigen (PSA) declines and delays of up to 150 days in PSA progression. Using a second generation PSMA CAR T cell, containing a ganciclovir induced suicide gene, in patients with chemotherapy pre-conditioning and no use of IL-2, there were two of seven patients with stable disease for more than 16 months, although no objective responses. ${ }^{50}$ This activity has led the investigators at Memorial Sloan Kettering to pursue another phase 1 trial of this construct that is currently in accrual (NCT01140373). Although the trial with the first generation CAR T cell had a higher response rate, the responses were more durable with the second generation CAR. It is not possible to discern the relative impact of first versus second generation CAR T cells directly due to the under-powered single arm study, as well as the impact of pre-conditioning with IL-2 therapy versus chemotherapy.

\section{Carboxy-anhydrase IX}

Carboxy-anhydrase-IX (CAIX) is frequently overexpressed on clear cell renal cell carcinoma (RCC) but also at low levels on bile duct epithelial cells. 12 patients with CAIX-expressing metastatic RCC were treated with a first-generation CAR targeting CAIX but the this led to significant liver toxicity requiring termination of the study. ${ }^{51}$ To prevent this toxicity, 4 patients were pre-treated with an anti-CAIX antibody and while this prevented the liver toxicity, there were no clinical responses demonstrated. 
However, CAIX is still being pursued as a target for adoptive dendritic cell therapy (see dendritic cell section).

\section{HPV-16 E6}

High-risk human papillomavirus (HPV) associated cancers include cervical, head and neck and penile cancer. In fact, approximately $30 \%$ of penile cancers test positive for HPV providing an obvious TAA for T-cell therapy targeting. Treatment with an HPV-16 E6 specific TCR is being tested in metastatic HPV associated cancers along with cyclophosphamide/fludarabine pre-conditioning and sequential high dose IL2. The study is closed to accrual and the results are awaited (NCT02280811).

\section{Other tumour associated antigens in urological malignancies}

Prostate stem cell antigen (PSCA) is highly expressed on primary and metastatic prostate cancer cells. ${ }^{52}$ A pre-clinical study using a third-generation CAR targeting PSCA with signalling domains of CD28 and OX-40, demonstrated significant delay in tumour growth rate and prolonged survival. ${ }^{53}$ Vascular endothelial growth factor 2 (VEGF-2) is overexpressed in a number of solid tumours including renal cancer and is associated with poor prognosis. ${ }^{54}$ The VEGFR-2 CAR-T cell has demonstrated promising pre-clinical activity in mouse models and is being investigated in a phase 1 study of RCC and melanoma (NCT01218867). Bladder cancer, has significant overexpression of a number of TAAs including NY-ESO-1, MAGE and BAGE ${ }^{55}$ for which T-cells programs are planned or ongoing.

\section{TIL Targets}

For successful TIL therapy a tumour must express a neoantigen that binds strongly to the MHC/TCR complex. The number of neoantigens correlates with the mutational burden of the tumour. Bladder cancer is the urological malignancy with the highest mutational burden, ${ }^{56}$ and like with melanoma and lung cancer, mutational load in bladder cancer correlates with response to checkpoint inhibition. For example, in a phase 2 study of the PD-L1 inhibitor atezolizumab, in 150 patients with advanced transitional cell carcinoma of the bladder, estimated mutational load was significantly higher in responders compared to non-responders (12.4 vs 6.4 per $\mathrm{Mb}, \mathrm{p}<0.0001){ }^{57}$ Bladder cancer would therefore be a potential tumour type to test both TIL therapy or adoptive dendritic cell therapies. 
Renal cell carcinoma (RCC) is considered an immunotherapy sensitive tumour although the mutational burden is low. ${ }^{7,56}$ A number of studies of TIL therapy have included RCC patients and early studies suggested response rates of up to $35 \%,{ }^{58}$ although from these small single arm trials and it was unclear whether the response was due to the concomitant IL-2 therapy or the TILs. Subsequently a phase III study in 178 RCC patients randomised to receive TIL and IL-2 or IL2 did not show benefit for the addition of TILs. However, only 39 patients went on receive TIL in the therapeutic arm, in part due to TIL production failure, which led to early closure of the study and therefore insufficient power to show a difference. ${ }^{59}$ Unlike many other tumours that have a high inflammatory infiltrate correlating with mutational burden, renal tumours often have a dense inflammatory infiltrate despite a low number of mutations. Presumably, RCC has a small number of strongly immunogenic antigens - either TAAs or neoantigens. Interestingly a recent report has suggested that inactivation of von Hippel-Lindau gene, the most common mutation in clear cell renal cell carcinoma, leads to selective expression of an endogenous retrovirus which in turn results in a highly immunogenic neoantigen, ${ }^{60}$ but this observation needs to be confirmed in independent series. Similarly, penile cancer often has dense inflammatory infiltrate on the background of relatively low mutational load. This can be partly explained by the highly immunogenic HPV induced neoepitopes but the remaining neoantigen repertoire is relatively understudied and could provide important insights about cellular therapy options for this difficult to treat disease.

Prostate cancer generally has a low mutational and neoepitope burden. ${ }^{56}$ While there are a relatively low number of TILs found in prostate cancer specimens, approximately $20 \%$ of high-risk localised prostate cancer patients have a significant infiltrate within the prostate (unpublished data). In the absence of agents causing migration of T-cells into the prostate, efforts for T-cell therapies may be most suited to this high infiltrate group. However, approximately $5 \%$ of patients with prostate cancer will have mismatch repair deficits, ${ }^{61}$ leading to a hypermutational state. In a single patient with MMR at our institution who underwent multiregional biopsies, the mutational burden was predominantly clonal (unpublished data) and therefore, based on the clonal neoantigen data presented earlier, we would hypothesise that they would respond to neoantigen directed dendritic or T-cell therapy.

\section{BEYOND $\alpha \beta$ T-CELLS}


In addition to the adaptive and innate defense mechanisms, there are unconventional T cells, for example gamma delta ( $\mathrm{\gamma} \delta$ ) T lymphocytes and natural killer T (NKT) cells. These cell types functionally and phenotypically cross over between the innate and the adaptive immune system.

\section{$\gamma \delta-\mathrm{T}$ cells}

Human $y \delta$ T cells comprise about $1-5 \%$ of the total T-cell population in the peripheral circulation but make up a major subset (up to $50 \%$ ) of some mucosal sites. $ү \delta$ T cells are involved in combating infectious diseases and have non-redundant inhibitory function preventing tumour development and progression (for review see ${ }^{62}$ ). Unlike $\alpha \beta$-T-cells, $ү \delta$-T cells do not require MHC class I and II for recognition of antigens and their selectivity is driven through a variable $(\mathrm{V})$ region of the TCR as opposed to the entire rearranged TCR. $\mathrm{V} \gamma \mathrm{g} \delta 2$-T-cells are the most prominent $\gamma \delta-\mathrm{T}$ cells in the peripheral circulation and respond to non-peptide phosphoantigens such as isopentenyl pyrophosphate (IPP), the mevalonate metabolite commonly generated by microbials. V $\gamma 9 \mathrm{~V} \delta 2-\mathrm{T}$-cells may also be activated by nitrogen containing bisphosphonates, such as zoledronic acid, often used in the treatment of prostate cancer. Given the role of $\gamma \delta-T$ cells in the anti-tumoural $T$ cell response, and importantly the ability to recognise antigens independently of MHC-TCR interaction, several clinical studies of adoptively transferred $ү \delta-T$ cells in metastatic prostate cancer and renal cell carcinoma are currently underway. ${ }^{63}$

\section{Natural Killer T-cell}

Natural killer T (NK-T) cells represent a mixed population of cells with biological features of both natural killer cells and T cells and represent approximately $0.1 \%$ of the peripheral $\mathrm{T}$ cell population. ${ }^{64} \mathrm{NK}-\mathrm{T}$ cells typically co-express an $\alpha \beta \mathrm{T}$ cell receptor in addition to cell surface markers of NK cells, for example NK1.1, CD16 and CD56. Two main subtypes of NK-T cells have been described; type 1 NK-T cells typically have a more limited repertoire as compared with classical $\alpha \beta$ T cells whereas type 2 NK-T cells have a more diverse repertoire. Both subtypes possess the ability to recognise glycolipids presented by the antigen-presenting molecule CD1d, a MHC-Class I-like molecule, bridging the innate and adaptive immune systems to enhance the antitumour immune response. Following activation, NK-T cells can rapidly produce various cytokines including IL-2, IFN- $\gamma$, TNF- $\alpha$ and IL-4, promoting NK mediated elimination 
of $\mathrm{MHC}$ negative tumours and $\mathrm{CD} 8+$ mediated cytotoxicity of $\mathrm{MHC}$ positive tumours providing an attractive therapeutic option as adoptive cellular therapy in cancer. Given the important role of NK-T cells in both the adaptive and immune anti-tumoural immune response, several clinical trials of NK-T cells are currently ongoing, including a phase I trial of autologous NK-T cells in various solid tumours including prostate cancer (NCT01801852). 


\section{COMBINATION THERAPIES IN UROLOGICAL MALIGNANCIES}

The overarching aim of combination therapy is to enhance patient outcomes while trying to minimise toxicity. There are a limitless number of potential combinations when considering 2 or 3 agents and therefore combination approaches must be based on a firm mechanistic understanding. The broad strategies are to: 1) increase the antigen exposure, 2) use two separate strategies to target the same tumour liability, 3) enable migration of effector cells into the tumour microenvironment (TME), 4) potentiate proliferation and/or persistence of effector T-cells within the TME and 5) reduce immunosuppressive factors in the TME. Some of the ongoing attempts in urological malignancies are presented here (Figure 3).

The backbone to many of the immune oncology combinations with adoptive cell therapies will be the immune checkpoint inhibitors. As this new class of oncology therapies has made such impact on the treatment of solid tumours, including bladder cancer, renal cancer and more recently prostate cancer, it would be remiss not to mention some of the seminal papers here despite not being the focus of this review. In $\mathrm{mBC}$, inhibition of PD-1/PD-L1 immune checkpoint has demonstrated significant single agent activity with overall response rates of about $15-31 \%$, which is further enriched in some studies by PD-L1 or TIL status ${ }^{57,65-67}$. Atezolizumab and nivolumab, have been FDA approved for the treatment of bladder cancer, and durvalumab and pembrolizumab are undergoing the accelerated approval process. In 821 previously treated renal cell carcinoma patients, nivolumab improved overall survival ( 25.0 vs 19.6months; HR 0.73, p=0.002) and had decreased toxicity compared to the previous standard of care, everolimus. ${ }^{68}$ In prostate cancer, initial studies of PD-L1/PD1 checkpoint inhibition demonstrated limited responses, ${ }^{69,70}$ causing a lull in their clinical development for this indication., Potential explanations for this lack of activity compared to tumour types such as melanoma, lung and bladder cancer include a relatively low mutational burden, a paucity of tumour infiltrating lymphocytes and selection of patients with high disease burden that were heavily pre-treated. Furthermore, it is possible that any efficacy signal was obscured by the small sample sizes and/or sub-optimal toxicity management of immune toxicity in these early studies leading to inadequate exposure to checkpoint inhibitor. Two phase III randomised clinical trials of ipilimumab in mCRPC have demonstrated no benefit in overall survival. ${ }^{71,72}$ More specifically, Ipilimumab monotherapy at $10 \mathrm{mg} / \mathrm{kg}$ had significant toxicity including 2 treatment related deaths, ${ }^{71}$ and may have contributed to this lack of benefit. In combination with radiotherapy however, ipilimumab therapy led to a trend 
to a 1.2 month overall survival benefit (11.2 vs 10.0 months; HR $0.85, p=0.053)^{72}$ although this duration of benefit is of questionable clinically significance. More promising however, was the recently reported early signal of activity seen in 5/27 prostate cancer patients receiving a PD-1 inhibitor in combination with enzalutamide, a drug that has demonstrated PD-L1 upregulation in pre-clinical models ${ }^{73-75}$

Despite this encouraging activity of checkpoint inhibitor monotherapy in urological malignancies the majority of patients still fail to respond or have durable benefit. The use of primed adoptive cells to enhance selectivity for antigens in combination with checkpoint inhibition to unleash the T-cell activity is one way to attempt to broaden the responders to immunotherapy. This is based on pre-clinical studies of mice treated with adoptive T-cell therapy, where the addition of checkpoint inhibition led to increased infiltration of T-cells in the tumour via a cytokine driven mechanism. ${ }^{76}$ This potential synergy of adoptive TIL therapy and PD-1 is being tested clinically in bladder cancer and the combination of adoptive dendritic cell therapy and anti CTLA-4 or PD$\mathrm{L} 1$ is being tested in separate studies in prostate cancer (Table 3 ). Furthermore, mouse models have shown that the PD-1/PD-L1 pathway negatively regulates CAR-T cell function, ${ }^{77}$ and therefore the combination of CAR-T cell therapy with immune checkpoint blockade is a rational combination for consideration in urological malignancies.

Dual adoptive therapy combinations are also being investigated in clinical trials. One strategy is to use two different immune mechanisms against the same target with a view to enhancing immune surveillance and limiting immune editing and resistance. For example, a group at UCLA, are treating NY-ESO expressing advanced cancers, which would potentially include bladder and prostate cancer, with a combination of NYESO-1 TCR-engineered cells and NY-ESO-1 peptide pulsed dendritic cells (NCT01697527). The results of this technically challenging study could have a major impact on the design of future combination immunotherapy studies.

Irrespective of the enthusiasm for new agents as part of the immunotherapy revolution, the substantial evidence that conventional cytotoxic chemotherapy can augment the anti-tumour immune response should not be overlooked. For example in model systems gemcitabine, that is typically used in bladder cancer, increases dendritic cell dependent antigen presentation, ${ }^{78}$ causes up-regulation of $\mathrm{MHC} 1$ expression, ${ }^{79}$ leads to a relative increase in T-cells, ${ }^{80}$ and a decrease in the immunosuppressive Treg 
cells. ${ }^{81}$ Combination studies of gemcitabine and cisplatin with checkpoint inhibition are ongoing in metastatic TCC but gemcitabine is a good candidate for use in combination with adoptive cell therapy. Similarly, in a prostate cancer cell line xenograft model, CD8+ cells with engineered TCR against PSA, MUC-1 or CEA, responses were enhanced when administered in combination with docetaxel, ${ }^{82}$ the most common chemotherapy given to prostate cancer patients. Combinations of docetaxel with dendritic cell therapy are being actively being pursued in prostate cancer (Table 3). Further work will inform on dose and schedules of chemotherapy in combination with adoptive cell therapy so that there is a correct balance between immunomodulation and lymphodepletion by the cytotoxic agent.

Hormonal agents are the mainstay of treating advanced prostate cancers and they too may be immunomodulatory. Initial studies in castrated mice demonstrated that the hormone deprivation led to increased T-cell number in lymphoid tissue and proliferation in response to antigen presentation. ${ }^{83} \mathrm{~A}$ recent murine study tested $2^{\text {nd }}$ generation hormone agents as immune adjuncts and reported that castration and abiraterone, a steroid synthesis inhibitor, treatment did not have a significant impact on CpG induced immune stimulation but enzalutamide, an androgen receptor antagonist, actually diminished the response.$^{84}$ However, recent cell line and patient data have suggested that enzalutamide resistant prostate tumours may express high levels of PD-L1 and respond to sipuleucel-T and PD-1 inhibition. ${ }^{73}$ Although conflicting, this preliminary data has encouraged investigators to test dendritic cell therapies (and checkpoint inhibitors) in combination with androgen deprivation therapy (Table 3).

Radiotherapy (RT) is an important immune modulator causing increased antigen presentation, elevated production of immunostimulatory cytokines, ${ }^{85}$ and enhanced effector T-cell and NK cell activity. ${ }^{86,87}$ There are a number of clinical trials combining cellular therapy and RT ongoing in urological malignancies, with both external beam $\mathrm{RT}$ and radium223 (Table 3).

In addition to their function as signalling components in transforming oncogenes, many protein kinases also play a role in the immune response ${ }^{88}$ and their inhibition has been linked to an enhanced immune response. In model systems, sunitinib, a multi-targeted tyrosine kinase inhibitor used as a first line therapy in $\mathrm{mRCC}$ and cabozantinib, a TKI predominantly targeting MET, RET and VEGFR-2 with activity in $\mathrm{mRCC}$ and $\mathrm{mCRPC}$ led to increased effector/regulator T-cell ratio (increased CD8+ cells and decreased TReg cells and MDSCs), ${ }^{89-91}$ which is a measure of immune 
surveillance. Both of these agents have also been reported to decrease the tumour vascularity thereby improving perfusion and immune cell access within the tumour microenvironment. ${ }^{92}$ These immune and microenvironment properties as well as direct modification of tumour cells would suggest that they are ideal agents to combine as part of immunotherapy combinations.

As previously mentioned, the results of the combination of the dendritic cell vaccine, AGS-003, in combination with sunitinib in patients with $\mathrm{mRCC}$ are eagerly awaited but on a cautionary note, the addition of two active monotherapies, IMA901 vaccine in combination with sunitinib failed to demonstrate an overall survival benefit compared with sunitinib alone, ${ }^{93}$. Unexpectedly, CD8+ specific T cell responses to IMA901 vaccine were markedly lower than in the positive phase 1 and 2 trials ${ }^{14}$ suggesting that the multi-targeted kinase inhibitor had a negative impact on vaccine responses and that our mechanistic understanding of this process is still in its infancy. Furthermore combinatorial toxicities may limit dual therapy usage such as the dose limiting hepatotoxicity in $\mathrm{mRCC}$ patients treated with nivolumab and pazopanib (another TKI used in $\mathrm{mRCC}$ ) arm of a dose escalation phase 1 trial. ${ }^{94}$ Further basic research to help underpin future rationale combination immune-oncology paradigms are required.

\section{CONCLUSIONS}

There is a need for improved therapies for advanced urological malignancies. This contemporary era of immunotherapy enlightenment presents vast opportunities to make inroads into controlling or even curing some urological cancers. Technological advancements in sequencing, informatics and high throughput avidity sorting of T-cells has helped to identify sub-groups of patients that might benefit from adoptive cell therapies, vaccines or checkpoint inhibitors. Dendritic cell therapies are showing considerable promise, particularly in prostate cancer, but there is a need to convert the responses into durable remissions. The significant improvements in gene transfer technology and ability to generate clinical grade cell products has enabled the development of therapeutic CAR-T cells, genetically engineered TCR T cells, adoptive dendritic and NK cells. However, these treatments will not work for everyone. The selection of patients, optimal sequencing and combination with novel and conventional therapies are key in improving patients' outcomes. These are expensive therapies that 
are sometimes challenging to deliver which may limit patient access to these treatments. To provide benefit to society therefore, immunotherapy will need to be set a high bar including durable remissions. This will only be acheived through a careful understanding of immune biology coupled with well-designed translational clinical studies allowing the optimism surrounding immunotherapy to be converted into improved patient outcomes in urological cancers.

\section{Acknowledgements}

This work was supported by the National Institute for Health Research, the University College London Hospitals Biomedical Research Centre and the Cancer Research UK (CRUK) University College London (UCL) Experimental Cancer Medicine Centre. M.L. is supported by CRUK, Prostate Cancer Foundation, Rosetrees Trust and an International Immuno-Oncology Network (II-ON) Bristol-Myers Squibb grant. S.A.Q. is a CRUK Senior Fellow and is funded by a CRUK Career Development Fellowship, the Cancer Research Institute Investigator Award and a CRUK Biotherapeutic Program Grant. K.S.P. receives funding from the NIHR BTRU for Stem Cells and Immunotherapies, of which he is the Scientific Director. Y.N.S.W is funded by a CRUK clinical fellowship grant. K.J. is funded by the Sam Keen Foundation and UCL/Royal Marsden Hospital NIHR Biomedical Research Centres. C.S. is Royal Society Napier Research Professor and is funded by Cancer Research UK (TRACERX), the CRUK Lung Cancer Centre of Excellence, Stand Up 2 Cancer (SU2C), the Rosetrees Trust, NovoNordisk Foundation (ID 16584), the Prostate Cancer Foundation, the Breast Cancer Research Foundation (BCRF), the European Research Council (THESEUS). This work was undertaken at UCL Hospitals/UCL with support from the CRUK-UCL Centre, the Cancer Immunotherapy Accelerator Award (CITA-CRUK) and The Khoo Teck Puat UK Foundation. This work was also supported by the Francis Crick Institute which receives its core funding from Cancer Research UK (FC001169), the UK Medical Research Council (FC001169), and the Wellcome Trust (FC001169); by the UK Medical Research Council (grant reference MR/FC001169/1). The funders had no role in the decision to submit, or preparation of the manuscript. 


\section{Search strategy and selection criteria}

We searched for articles on PubMed published in English between August 15, 2011 , and August 15, 2016, with combination of the following terms: "genitourinary malignancies", "urological malignancies", "prostate cancer", "kidney cancer", "bladder cancer", "penile cancer", "neoantigens", "cellular therapies", "dendritic cell therapy", "TIL therapy", "natural killer cell therapy", "CAR-T cell therapy", "combination". Abstracts from major oncology conferences, including the American Association for Cancer Research, the American Society of Clinical Oncology and the European Society for Medical Oncology were also searched. The authors' individual libraries were also accessed.

\section{Contributors}

Article conception, literature search and formation by M.L. Y.N.S.W and K.J with equal contribution to all sections of the article including figures and illustrations. C.S., K.P and S.A.Q edited and contributed to the TIL and dendritic section writing. M.P contributed to the CAR-T cell section writing.

\section{Declaration of interests}

C.S reports personal fees from Roche, Pfizer, Boehringer Ingelheim, Novartis, Celgene, Servier, Eli Lily, and from GlaxoSmithKline, outside of the submitted work; and stock options from Achilles Therapeutics, Epic Biosciences, Grail and Apogen Biotech. S.A.Q reports stock options from Achilles Therapeutics. M.P reports grants from Cellectis, Roche, Amgen outside of the submitted work; and stock options from Autolus Ltd and UCL for patents invented, including CAR-T cell constructs. The other authors declared no conflicts of interest. 


\section{REFERENCES}

1. Coley WB. The treatment of malignant tumors by repeated inoculations of erysipelas. With a report of ten original cases. 1893. Clin Orthop Relat Res 1991; (262): 3-11.

2. Rosenberg SA, Lotze MT, Muul LM, et al. Observations on the systemic administration of autologous lymphokine-activated killer cells and recombinant interleukin-2 to patients with metastatic cancer. The New England journal of medicine 1985; 313(23): 1485-92.

3. Tran E, Robbins PF, Lu YC, et al. T-Cell Transfer Therapy Targeting Mutant KRAS in Cancer. N Engl J Med 2016; 375(23): 2255-62.

4. Shortman K, Liu YJ. Mouse and human dendritic cell subtypes. Nat Rev Immunol 2002; 2(3): 151-61.

5. Fearnley DB, Whyte LF, Carnoutsos SA, Cook AH, Hart DN. Monitoring human blood dendritic cell numbers in normal individuals and in stem cell transplantation. Blood 1999; 93(2): 728-36.

6. Kantoff PW, Higano CS, Shore ND, et al. Sipuleucel-T immunotherapy for castration-resistant prostate cancer. The New England journal of medicine 2010; 363(5): 411-22.

7. Motzer RJ, Escudier B, McDermott DF, et al. Nivolumab versus Everolimus in Advanced Renal-Cell Carcinoma. The New England journal of medicine 2015; 373(19): 1803-13.

8. Zhao J, Xu W, Zhang Z, et al. Prognostic role of HER2 expression in bladder cancer: a systematic review and meta-analysis. International urology and nephrology 2015; 47(1): 87-94.

9. Bajorin DF, Sharma P, Quinn DI, et al. Phase 2 trial results of DN24-02, a HER2-targeted autologous cellular immunotherapy in HER2+ urothelial cancer patients (pts). J Clin Oncol 2016; 34(suppl; abstr 4513).

10. Podrazil M, Horvath R, Becht E, et al. Phase I/II clinical trial of dendriticcell based immunotherapy (DCVAC/PCa) combined with chemotherapy in patients with metastatic, castration-resistant prostate cancer. Oncotarget 2015; 6(20): 18192-205.

11. Amin A, Dudek AZ, Logan TF, et al. Survival with AGS-003, an autologous dendritic cell-based immunotherapy, in combination with sunitinib in unfavorable risk patients with advanced renal cell carcinoma (RCC): Phase 2 study results. J Immunother Cancer 2015; 3: 14.

12. Li D, Romain G, Flamar AL, et al. Targeting self- and foreign antigens to dendritic cells via DC-ASGPR generates IL-10-producing suppressive CD4+ T cells. J Exp Med 2012; 209(1): 109-21.

13. Kantoff PW, Gulley JL, Pico-Navarro C. Revised Overall Survival Analysis of a Phase II, Randomized, Double-Blind, Controlled Study of PROSTVAC in Men With Metastatic Castration-Resistant Prostate Cancer. J Clin Oncol 2016.

14. Walter S, Weinschenk T, Stenzl A, et al. Multipeptide immune response to cancer vaccine IMA901 after single-dose cyclophosphamide associates with longer patient survival. Nat Med 2012; 18(8): 1254-61. 
15. Obara W, Eto M, Mimata $\mathrm{H}$, et al. A phase I/II study of cancer peptide vaccine S-288310 in patients with advanced urothelial carcinoma of the bladder. Ann Oncol 2016.

16. van Rooij N, van Buuren MM, Philips D, et al. Tumor exome analysis reveals neoantigen-specific $\mathrm{T}$-cell reactivity in an ipilimumab-responsive melanoma. Journal of clinical oncology : official journal of the American Society of Clinical Oncology 2013; 31(32): e439-42.

17. Robbins PF, Lu YC, El-Gamil M, et al. Mining exomic sequencing data to identify mutated antigens recognized by adoptively transferred tumor-reactive $\mathrm{T}$ cells. Nature medicine 2013; 19(6): 747-52.

18. Snyder A, Makarov V, Merghoub T, et al. Genetic basis for clinical response to CTLA-4 blockade in melanoma. The New England journal of medicine 2014; 371(23): 2189-99.

19. McGranahan N, Furness AJ, Rosenthal R, et al. Clonal neoantigens elicit T cell immunoreactivity and sensitivity to immune checkpoint blockade. Science 2016; 351(6280): 1463-9.

20. Morvan MG, Lanier LL. NK cells and cancer: you can teach innate cells new tricks. Nature reviews Cancer 2016; 16(1): 7-19.

21. Sabry M, Tsirogianni M, Bakhsh IA, et al. Leukemic priming of resting NK cells is killer Ig-like receptor independent but requires CD15-mediated CD2 ligation and natural cytotoxicity receptors. Journal of immunology 2011; 187(12): 6227-34.

22. Kottaridis PD, North J, Tsirogianni M, et al. Two-Stage Priming of Allogeneic Natural Killer Cells for the Treatment of Patients with Acute Myeloid Leukemia: A Phase I Trial. PLoS One 2015; 10(6): e0123416.

23. Hu W, Zheng RR, Cui HX, Yue D, Wang Y, Jiang YH. Effects of bortezomib in sensitizing human prostate cancer cell lines to NK-mediated cytotoxicity. Asian J Androl 2012; 14(5): 695-702.

24. Arai $S$, Meagher $\mathrm{R}$, Swearingen $\mathrm{M}$, et al. Infusion of the allogeneic cell line NK-92 in patients with advanced renal cell cancer or melanoma: a phase I trial. Cytotherapy 2008; 10(6): 625-32.

25. Southam CM, Brunschwig A, Levin AG, Dizon QS. Effect of leukocytes on transplantability of human cancer. Cancer 1966; 19(11): 1743-53.

26. Kolb HJ, Mittermuller J, Clemm C, et al. Donor leukocyte transfusions for treatment of recurrent chronic myelogenous leukemia in marrow transplant patients. Blood 1990; 76(12): 2462-5.

27. Rosenberg SA, Packard BS, Aebersold PM, et al. Use of tumor-infiltrating lymphocytes and interleukin-2 in the immunotherapy of patients with metastatic melanoma. A preliminary report. The New England journal of medicine 1988; 319(25): 1676-80.

28. Besser MJ, Shapira-Frommer R, Schachter J. Tumor-Infiltrating Lymphocytes: Clinical Experience. Cancer J 2015; 21(6): 465-9.

29. Goedegebuure PS, Douville LM, Li H, et al. Adoptive immunotherapy with tumor-infiltrating lymphocytes and interleukin-2 in patients with metastatic malignant melanoma and renal cell carcinoma: a pilot study. J Clin Oncol 1995; 13(8): 1939-49.

30. Baldan V, Griffiths R, Hawkins RE, Gilham DE. Efficient and reproducible generation of tumour-infiltrating lymphocytes for renal cell carcinoma. Br J Cancer 2015; 112(9): 1510-8. 
31. Klebanoff CA, Rosenberg SA, Restifo NP. Prospects for gene-engineered T cell immunotherapy for solid cancers. Nat Med 2016; 22(1): 26-36.

32. Chekmasova AA, Rao TD, Nikhamin Y, et al. Successful Eradication of Established Peritoneal Ovarian Tumors in SCID-Beige Mice following Adoptive Transfer of T Cells Genetically Targeted to the MUC16 Antigen. Clinical Cancer Research 2010; 16(14): 3594-606.

33. Lamers $\mathrm{CH}$, Sleijfer S, Vulto AG, et al. Treatment of metastatic renal cell carcinoma with autologous T-lymphocytes genetically retargeted against carbonic anhydrase IX: first clinical experience. Journal of clinical oncology : official journal of the American Society of Clinical Oncology 2006; 24(13): e20-2.

34. Till BG, Jensen MC, Wang J, et al. Adoptive immunotherapy for indolent non-Hodgkin lymphoma and mantle cell lymphoma using genetically modified autologous CD20-specific T cells. Blood 2008; 112(6): 2261-71.

35. Finney HM, Lawson AD, Bebbington CR, Weir AN. Chimeric receptors providing both primary and costimulatory signaling in T cells from a single gene product. Journal of immunology 1998; 161(6): 2791-7.

36. Pule MA, Straathof KC, Dotti G, Heslop HE, Rooney CM, Brenner MK. A chimeric $\mathrm{T}$ cell antigen receptor that augments cytokine release and supports clonal expansion of primary human T cells. Mol Ther 2005; 12(5): 933-41.

37. Savoldo B, Rooney CM, Di Stasi A, et al. Epstein Barr virus specific cytotoxic T lymphocytes expressing the anti-CD30zeta artificial chimeric T-cell receptor for immunotherapy of Hodgkin disease. Blood 2007; 110(7): 2620-30.

38. Ghorashian S, Pule M, Amrolia P. CD19 chimeric antigen receptor T cell therapy for haematological malignancies. Br J Haematol 2015; 169(4): 463-78.

39. Grupp SA, Kalos M, Barrett D, et al. Chimeric antigen receptor-modified T cells for acute lymphoid leukemia. The New England journal of medicine 2013; 368(16): 1509-18.

40. Kershaw MH, Westwood JA, Darcy PK. Gene-engineered T cells for cancer therapy. Nat Rev Cancer 2013; 13(8): 525-41.

41. Parkhurst MR, Yang JC, Langan RC, et al. T cells targeting carcinoembryonic antigen can mediate regression of metastatic colorectal cancer but induce severe transient colitis. Mol Ther 2011; 19(3): 620-6.

42. Stanislawski T, Voss RH, Lotz C, et al. Circumventing tolerance to a human MDM2-derived tumor antigen by TCR gene transfer. Nature immunology 2001; 2(10): 962-70.

43. Johnson LA, Morgan RA, Dudley ME, et al. Gene therapy with human and mouse $\mathrm{T}$-cell receptors mediates cancer regression and targets normal tissues expressing cognate antigen. Blood 2009; 114(3): 535-46.

44. Morgan RA, Chinnasamy N, Abate-Daga D, et al. Cancer regression and neurological toxicity following anti-MAGE-A3 TCR gene therapy. J Immunother 2013; 36(2): 133-51.

45. Robbins PF, Morgan RA, Feldman SA, et al. Tumor regression in patients with metastatic synovial cell sarcoma and melanoma using genetically engineered lymphocytes reactive with NY-ESO-1. J Clin Oncol 2011; 29(7): 917-24.

46. Kinoshita Y, Kuratsukuri K, Landas S, et al. Expression of prostate-specific membrane antigen in normal and malignant human tissues. World journal of surgery 2006; 30(4): 628-36.

47. Sacha P, Zamecnik J, Barinka C, et al. Expression of glutamate carboxypeptidase II in human brain. Neuroscience 2007; 144(4): 1361-72. 
48. Haffner MC, Kronberger IE, Ross JS, et al. Prostate-specific membrane antigen expression in the neovasculature of gastric and colorectal cancers. Human pathology 2009; 40(12): 1754-61.

49. Junghans RP, Ma Q, Rathore R, et al. Phase I Trial of Anti-PSMA Designer CAR-T Cells in Prostate Cancer: Possible Role for Interacting Interleukin 2-T Cell Pharmacodynamics as a Determinant of Clinical Response. The Prostate 2016; 76(14): 1257-70.

50. Slovin SF, Wang X, Hullings $M$, et al. Chimeric antigen receptor (CAR+) modified $\mathrm{T}$ cells targeting prostate-specific membrane antigen (PSMA) in patients (pts) with castrate metastatic prostate cancer (CMPC). J Clin Oncol 2013; 31(suppl 6; abstr 72).

51. Lamers $\mathrm{CH}$, Sleijfer S, van Steenbergen S, et al. Treatment of metastatic renal cell carcinoma with CAIX CAR-engineered T cells: clinical evaluation and management of on-target toxicity. Mol Ther 2013; 21(4): 904-12.

52. $\mathrm{Gu} \mathrm{Z}$, Thomas G, Yamashiro J, et al. Prostate stem cell antigen (PSCA) expression increases with high gleason score, advanced stage and bone metastasis in prostate cancer. Oncogene 2000; 19(10): 1288-96.

53. Hillerdal V, Ramachandran M, Leja J, Essand M. Systemic treatment with CAR-engineered $\mathrm{T}$ cells against PSCA delays subcutaneous tumor growth and prolongs survival of mice. BMC Cancer 2014; 14: 30.

54. Hanahan D, Weinberg Robert A. Hallmarks of Cancer: The Next Generation. Cell; 144(5): 646-74.

55. Fradet Y, Picard V, Bergeron A, LaRue H. Cancer-testis antigen expression in bladder cancer. Progres en urologie : journal de l'Association francaise d'urologie et de la Societe francaise d'urologie 2005; 15(6 Suppl 1): 1303-13.

56. Alexandrov LB, Nik-Zainal S, Wedge DC, et al. Signatures of mutational processes in human cancer. Nature 2013; 500(7463): 415-21.

57. Rosenberg JE, Hoffman-Censits J, Powles T, et al. Atezolizumab in patients with locally advanced and metastatic urothelial carcinoma who have progressed following treatment with platinum-based chemotherapy: a single-arm, multicentre, phase 2 trial. Lancet 2016.

58. Figlin RA, Pierce WC, Kaboo R, et al. Treatment of metastatic renal cell carcinoma with nephrectomy, interleukin-2 and cytokine-primed or CD8(+) selected tumor infiltrating lymphocytes from primary tumor. J Urol 1997; 158(3 Pt 1): 740-5.

59. Figlin RA, Thompson JA, Bukowski RM, et al. Multicenter, randomized, phase III trial of CD8(+) tumor-infiltrating lymphocytes in combination with recombinant interleukin-2 in metastatic renal cell carcinoma. J Clin Oncol 1999; 17(8): 2521-9.

60. Cherkasova E, Malinzak E, Rao S, et al. Inactivation of the von HippelLindau tumor suppressor leads to selective expression of a human endogenous retrovirus in kidney cancer. Oncogene 2011; 30(47): 4697-706.

61. Pritchard CC, Morrissey C, Kumar A, et al. Complex MSH2 and MSH6 mutations in hypermutated microsatellite unstable advanced prostate cancer. Nature communications 2014; 5: 4988.

62. Silva-Santos B, Serre K, Norell H. gammadelta T cells in cancer. Nat Rev Immunol 2015; 15(11): 683-91.

63. Kobayashi H, Tanaka Y, Yagi J, Minato N, Tanabe K. Phase I/II study of adoptive transfer of gammadelta T cells in combination with zoledronic acid and 
IL-2 to patients with advanced renal cell carcinoma. Cancer Immunol Immunother 2011; 60(8): 1075-84.

64. Godfrey DI, MacDonald HR, Kronenberg M, Smyth MJ, Van Kaer L. NKT cells: what's in a name? Nat Rev Immunol 2004; 4(3): 231-7.

65. Powles T, Eder JP, Fine GD, et al. MPDL3280A (anti-PD-L1) treatment leads to clinical activity in metastatic bladder cancer. Nature 2014; 515(7528): 558-62.

66. Massard C, Gordon MS, Sharma S, et al. Safety and Efficacy of Durvalumab (MEDI4736), an Anti-Programmed Cell Death Ligand-1 Immune Checkpoint Inhibitor, in Patients With Advanced Urothelial Bladder Cancer. Journal of clinical oncology: official journal of the American Society of Clinical Oncology 2016; 34(26): 3119-25.

67. Sharma P, Retz M, Siefker-Radtke A, et al. Nivolumab in metastatic urothelial carcinoma after platinum therapy (CheckMate 275): a multicentre, single-arm, phase 2 trial. The Lancet Oncology 2017.

68. Motzer RJ, Escudier B, McDermott DF, et al. Nivolumab versus Everolimus in Advanced Renal-Cell Carcinoma. New England Journal of Medicine 2015; 373(19): 1803-13.

69. Topalian SL, Hodi FS, Brahmer JR, et al. Safety, activity, and immune correlates of anti-PD-1 antibody in cancer. The New England journal of medicine 2012; 366(26): 2443-54.

70. Taube JM, Klein A, Brahmer JR, et al. Association of PD-1, PD-1 ligands, and other features of the tumor immune microenvironment with response to anti-PD1 therapy. Clinical cancer research : an official journal of the American Association for Cancer Research 2014; 20(19): 5064-74.

71. Beer TM, Kwon ED, Drake CG, et al. Randomized, Double-Blind, Phase III Trial of Ipilimumab Versus Placebo in Asymptomatic or Minimally Symptomatic Patients With Metastatic Chemotherapy-Naive Castration-Resistant Prostate Cancer. Journal of clinical oncology : official journal of the American Society of Clinical Oncology 2017; 35(1): 40-7.

72. Kwon ED, Drake CG, Scher HI, et al. Ipilimumab versus placebo after radiotherapy in patients with metastatic castration-resistant prostate cancer that had progressed after docetaxel chemotherapy (CA184-043): a multicentre, randomised, double-blind, phase 3 trial. The lancet oncology 2014; 15(7): 700-12. 73. Graff JN, Alumkal JJ, Drake CG, et al. Early evidence of anti-PD-1 activity in enzalutamide-resistant prostate cancer. Oncotarget 2016.

74. Bishop JL, Sio A, Angeles A, et al. PD-L1 is highly expressed in Enzalutamide resistant prostate cancer. Oncotarget 2015; 6(1): 234-42.

75. Graff JN, Alumkal JJ, Drake CG, et al. First evidence of significant clinical activity of PD-1 inhibitors in metastatic, castration resistant prostate cancer (mCRPC). Annals of Oncology 2016; 27(Suppl 6): 7190.

76. Peng $\mathrm{W}$, Liu $\mathrm{C}, \mathrm{Xu} \mathrm{C}$, et al. PD-1 blockade enhances T-cell migration to tumors by elevating IFN-gamma inducible chemokines. Cancer research 2012; 72(20): 5209-18.

77. Cherkassky L, Morello A, Villena-Vargas J, et al. Human CAR T cells with cell-intrinsic PD-1 checkpoint blockade resist tumor-mediated inhibition. J Clin Invest 2016; 126(8): 3130-44.

78. Nowak AK, Lake RA, Marzo AL, et al. Induction of tumor cell apoptosis in vivo increases tumor antigen cross-presentation, cross-priming rather than crosstolerizing host tumor-specific CD8 T cells. J Immunol 2003; 170(10): 4905-13. 
79. Liu WM, Fowler DW, Smith P, Dalgleish AG. Pre-treatment with chemotherapy can enhance the antigenicity and immunogenicity of tumours by promoting adaptive immune responses. Br J Cancer 2010; 102(1): 115-23.

80. Nowak AK, Robinson BW, Lake RA. Gemcitabine exerts a selective effect on the humoral immune response: implications for combination chemoimmunotherapy. Cancer research 2002; 62(8): 2353-8.

81. Shevchenko I, Karakhanova S, Soltek S, et al. Low-dose gemcitabine depletes regulatory $\mathrm{T}$ cells and improves survival in the orthotopic Panc02 model of pancreatic cancer. International journal of cancer 2013; 133(1): 98-107.

82. Hodge JW, Garnett CT, Farsaci B, et al. Chemotherapy-induced immunogenic modulation of tumor cells enhances killing by cytotoxic $\mathrm{T}$ lymphocytes and is distinct from immunogenic cell death. International journal of cancer 2013; 133(3): 624-36.

83. Roden AC, Moser MT, Tri SD, et al. Augmentation of T cell levels and responses induced by androgen deprivation. Journal of immunology 2004; 173(10): 6098-108.

84. $\mathrm{Pu} Y, \mathrm{Xu} \mathrm{M}$, Liang Y, et al. Androgen receptor antagonists compromise T cell response against prostate cancer leading to early tumor relapse. Science translational medicine 2016; 8(333): 333ra47.

85. Lugade AA, Sorensen EW, Gerber SA, Moran JP, Frelinger JG, Lord EM. Radiation-induced IFN-gamma production within the tumor microenvironment influences antitumor immunity. Journal of immunology (Baltimore, Md : 1950) 2008; 180(5): 3132-9.

86. Lee Y, Auh SL, Wang Y, et al. Therapeutic effects of ablative radiation on local tumor require CD8+ T cells: changing strategies for cancer treatment. Blood 2009; 114(3): 589-95.

87. Takeshima T, Chamoto K, Wakita D, et al. Local radiation therapy inhibits tumor growth through the generation of tumor-specific CTL: its potentiation by combination with Th1 cell therapy. Cancer research 2010; 70(7): 2697-706.

88. Jiang Q, Weiss JM, Wiltrout RH. A matched couple: Combining kinase inhibitors with immunotherapy for cancer treatment. Oncoimmunology 2012; 1(1): 115-7.

89. Adotevi $\mathrm{O}$, Pere $\mathrm{H}$, Ravel $\mathrm{P}$, et al. A decrease of regulatory $\mathrm{T}$ cells correlates with overall survival after sunitinib-based antiangiogenic therapy in metastatic renal cancer patients. J Immunother 2010; 33(9): 991-8.

90. Farsaci B, Higgins JP, Hodge JW. Consequence of dose scheduling of sunitinib on host immune response elements and vaccine combination therapy. Int J Cancer 2012; 130(8): 1948-59.

91. Kwilas AR, Ardiani A, Donahue RN, Aftab DT, Hodge JW. Dual effects of a targeted small-molecule inhibitor (cabozantinib) on immune-mediated killing of tumor cells and immune tumor microenvironment permissiveness when combined with a cancer vaccine. J Transl Med 2014; 12: 294.

92. Farsaci B, Donahue RN, Coplin MA, et al. Immune consequences of decreasing tumor vasculature with antiangiogenic tyrosine kinase inhibitors in combination with therapeutic vaccines. Cancer Immunol Res 2014; 2(11): 1090102.

93. Rini BI, Stenzl A, Zdrojowy R, et al. IMA901, a multipeptide cancer vaccine, plus sunitinib versus sunitinib alone, as first-line therapy for advanced or metastatic renal cell carcinoma (IMPRINT): a multicentre, open-label, 
randomised, controlled, phase 3 trial. The Lancet Oncology 2016; 17(11): 1599611.

94. Amin A, Plimack ER, Infante JR, et al. Nivolumab (anti-PD-1; BMS-936558, ONO-4538) in combination with sunitinib or pazopanib in patients (pts) with metastatic renal cell carcinoma (mRCC). Journal of clinical oncology : official journal of the American Society of Clinical Oncology 2014; 32 (suppl; abstract 5010). 